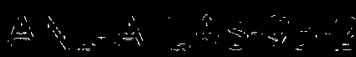
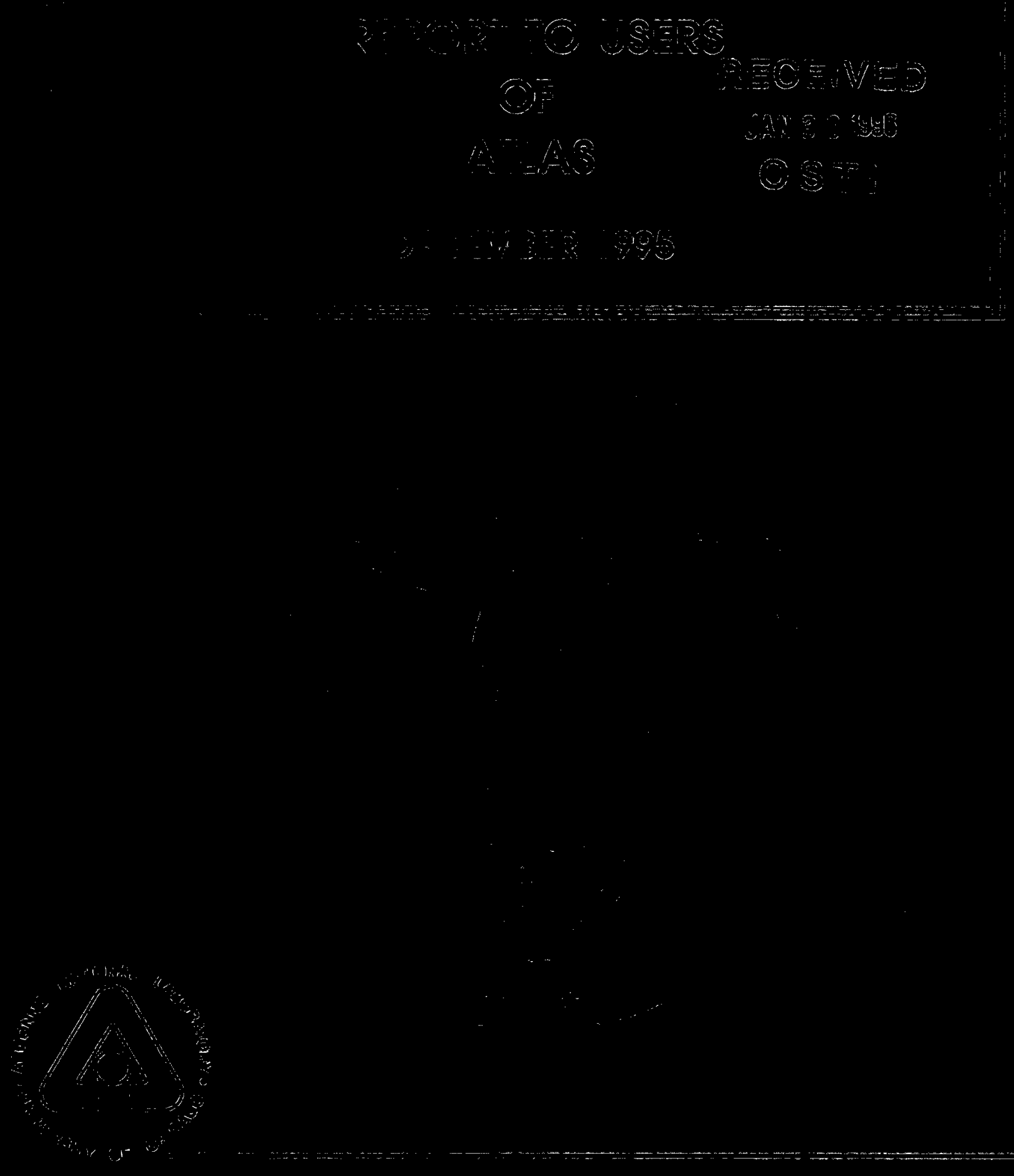

4.

w

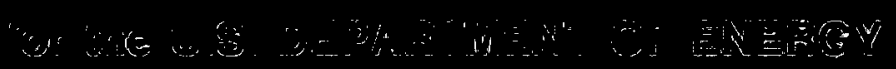

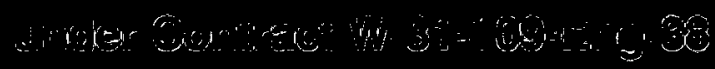


* in A. 堆 s)

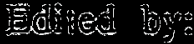

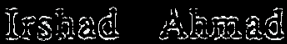 \\ 的通 \\ Brides CHe?
}

\begin{abstract}
$=\ldots$ a

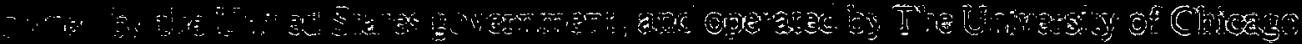

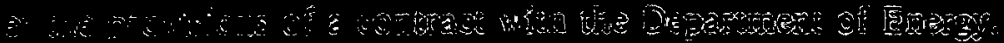

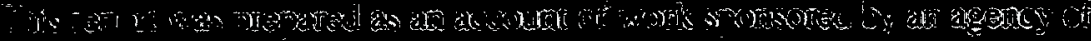

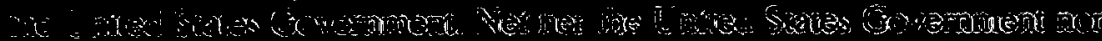
a

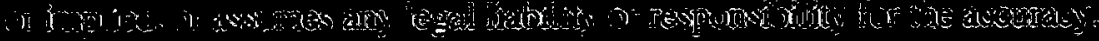

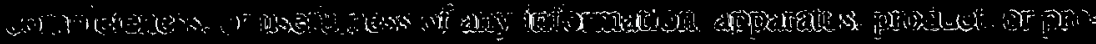

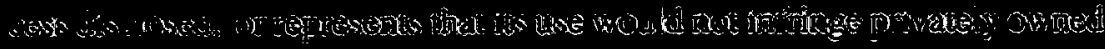
-g to s.

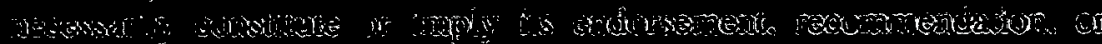

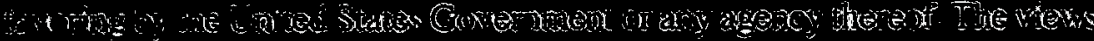

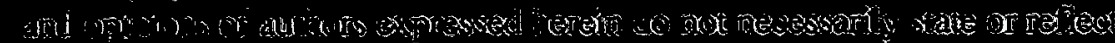

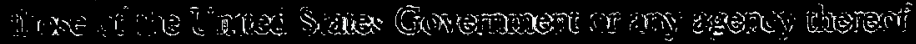




\title{
REPORT TO USERS OF ATLAS
}

\author{
December 1995
}

\section{Table of Contents}

I. Status of the ATLAS Accelerator

II. Highlights of Recent Research at ATLAS

III. Research Related to the Concept for an Advanced Exotic Beam

Facility Based on ATLAS

IV. Program Advisory Committee

V. ATLAS User Group Executive Committee

\section{STATUS OF THE ATLAS ACCELERATOR}

The ATLAS accelerator facility provided 4589 beam-hours of research time to users during the 1995 fiscal year. This was accomplished during an operating year shortened by 10.5 weeks for a major cryogenics system upgrade. The facility operated on a continuous 7-day/week schedule and provided beams of 29 different isotopes to the research program spanning the mass range from protons to uranium, more than any previous year in spite of the long downtime. Figure 1 shows the percentage of time ATLAS was used for each beam.

\section{ATLAS Beams for FY 1995}

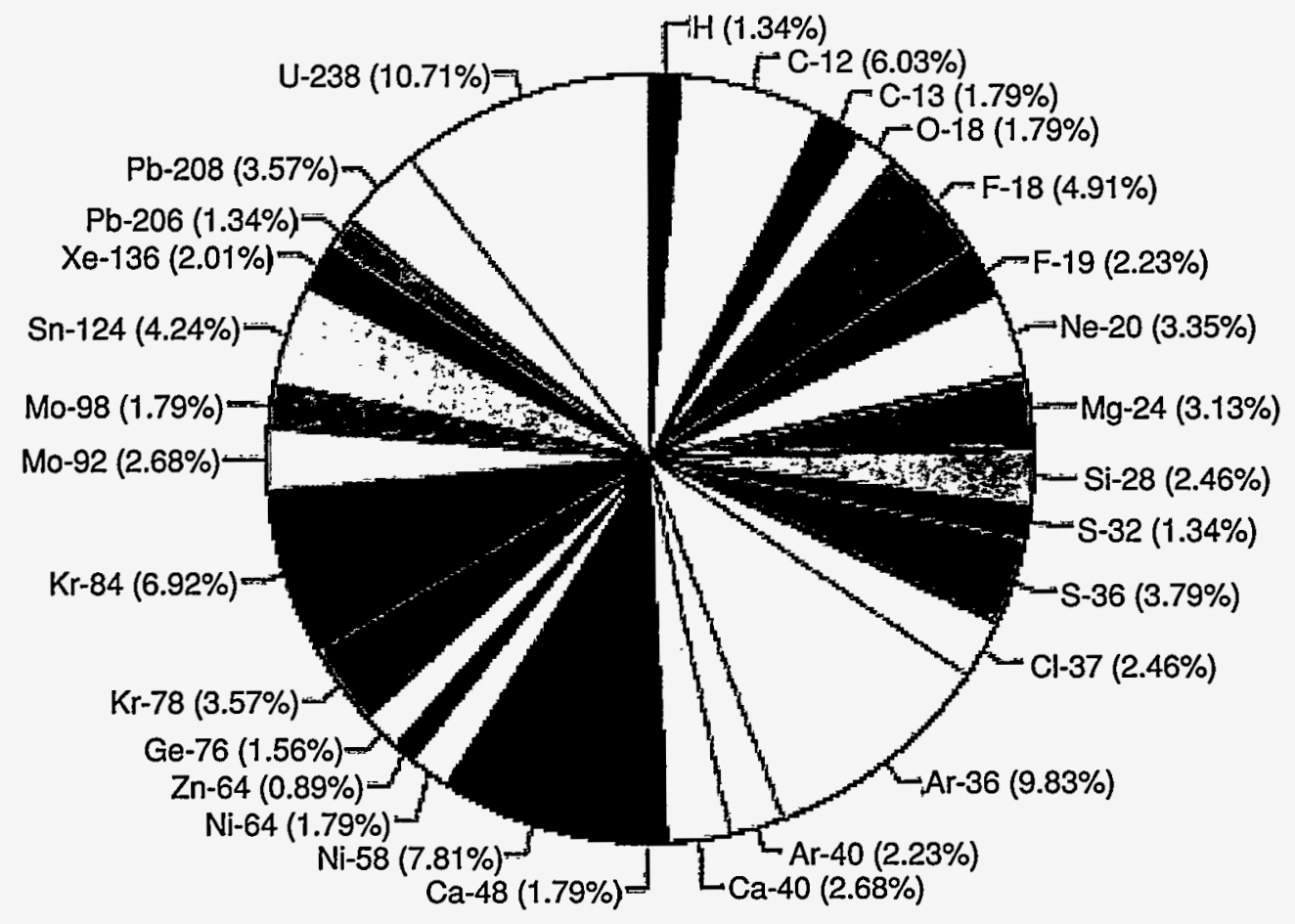

Fig. 1. Percentage of research time for each of the 29 isotopes provided to the experimental program in FY 1995. 
For the first time in recent memory, no experiments using 160 were scheduled which emphasizes the growing importance of heavier species in the research program.

A ten-week shutdown of the facility took place from April through June. The primary focus of the shutdown was the installation of two new wet expander engines in the cryogenic system to increase the system cooling capacity, efficiency and reliability. The installation went smoothly and the expected performance improvements appear to have been realized. Additional tests and measurements are planned to better quantify the improvements, but initial indications are that the system capacity has increased by approximately $25 \%$ and a similar improvement in energy efficiency has also occurred at a fixed capacity. Reduced electrical consumption and enhanced system reliability are both the direct benefits of the upgrade.

The new $14 \mathrm{GHz}$ ECR ion source project continues to move forward at a good pace. The most important development for the project in the past six months has been the arrival of Michael Schlapp, our new post-doc for ECR development. Michael received his Ph.D. from the University of Giessen, Germany where he led a group of people in constructing a number of successful ECR ion sources. Michael replaces Richard Harkewicz in the accelerator group, who returned to Michigan State University in May to lead the ECR group there.

The construction of the new building addition for the ECR ion source is essentially complete and procurement of parts and construction of the high-voltage platform on which the new source will be mounted has begun. Completion of the platform is scheduled for February 1,1996, at which time installation of the new source will commence.

A large fraction of the source components are now in hand, including the permanent magnet hexapole, the $14 \mathrm{GHz}$ transmitter, the solenoid coils, and the vacuum chamber. The hexapole magnet has been assembled and its field mapped. First plasma in the new source is expected to occur by May, 1996. The connecting beamline from the source to ATLAS is not fully funded in this fiscal year, but we expect first beam from the new source to be delivered to ATLAS for the research program by early 1997 . Figure 2 shows the new building addition at an earlier stage of construction (September, 1995).

\section{HIGHLIGHTS OF RECENT RESEARCH AT ATLAS}

Some highlights of recent experiments performed at ATLAS are summarized below. Figure 3 shows a photograph of an experimental group from the University of Surrey, U.K. which performed an experiment with the FMA.

\section{A. APEX PROGRESS REPORT}

(Argonne, Florida State, Michigan State, Queen's, Princeton, Rochester, Washington, and Yale)

A major milestone in the APEX collaboration was reached with the publication of the first results of searches for peaks in positron-electron sum energy spectra obtained in collisions of ${ }^{238} \mathrm{U}+{ }^{181} \mathrm{Ta}$ and ${ }^{238} \mathrm{U}+{ }^{232} \mathrm{Th}$ [1]. In these data, sharp sum energy lines reported previously $[2,3]$ were not observed. With a good understanding of the response of the APEX spectrometer obtained with radioactive sources, Monte-Carlo simulations 


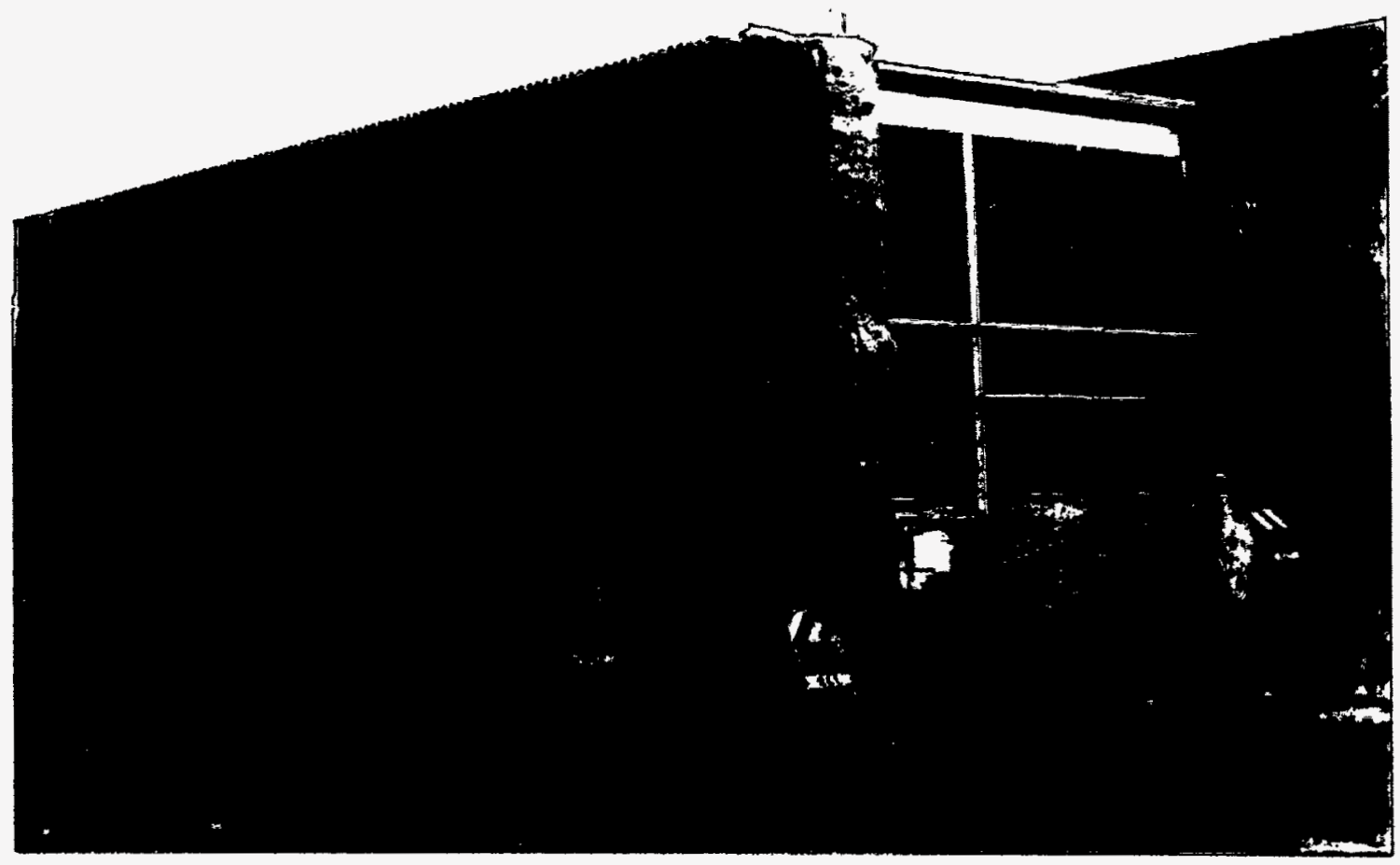

Fig. 2. Photograph of the ATLAS building addition for the new ECR ion source and its high-voltage platform under construction in September. The addition is now complete.

using the code GEANT, and, most importantly, an in-beam measurement of internal pair conversion (IPC) of the $1.84-\mathrm{MeV}$ transition in ${ }^{206} \mathrm{~Pb}$ (see previous newsletter), limits have been placed on the production cross section for particular scenarios for production of a sharp $\mathrm{e}^{+}-\mathrm{e}-$ sum energy line in the ${ }^{238} \mathrm{U}+{ }^{232} \mathrm{Th}$ system. For the specific case of a decaying light neutral particle, as suggested to account for the lines observed in ${ }^{238} \mathrm{U}+{ }^{232} \mathrm{Th}$ collisions [2,3], two analyses have been used to address this possibility. The results of an analysis of the coincidence data for the ${ }^{238} \mathrm{U}+{ }^{232} \mathrm{Th}$ system under the conditions of a socalled "wedge-cut" [2,3], and more restrictive cuts, which emphasize the sensitivity of the apparatus to particle-like $\mathrm{e}^{+}$-e- kinematics, appear in Fig. 4a. The histograms in Fig. 4 represent calculations of the uncorrelated $\mathrm{e}^{+}-\mathrm{e}^{-}$spectrum, obtained by mixing the energies of positrons and electrons from different events. The corresponding limits for the existence of a peak under these analysis conditions appear in Fig. $4 \mathrm{~b}$, and are on the order of a fraction of a microbarn/sr. For comparison, the values of the previously reported cross sections are given as the shaded bar in Fig. $4 \mathrm{~b}$. The current limits are approximately one order of magnitude below the cross sections previously reported, constituting a real disagreement between the present results and previous experiments.

While further searches for peaks have not been ruled out by the collaboration, the possibilities for experiments with other physics goals that can take advantage of the unique capabilities of the APEX spectrometer are being examined. Of particular interest in this regard is IPC. While the IPC measurements in ${ }^{206} \mathrm{~Pb}$ are certainly interesting, in order to test theoretical predictions of the absolute conversion coefficients and $\mathrm{e}^{+}-\mathrm{e}^{-}$angular correlations, quite useful information can be obtained from measurements with radioactive sources. One particularly interesting question involves a comparison of IPC from E1 and M1 transitions. While E1 conversion appears to be quite well understood, considerable 


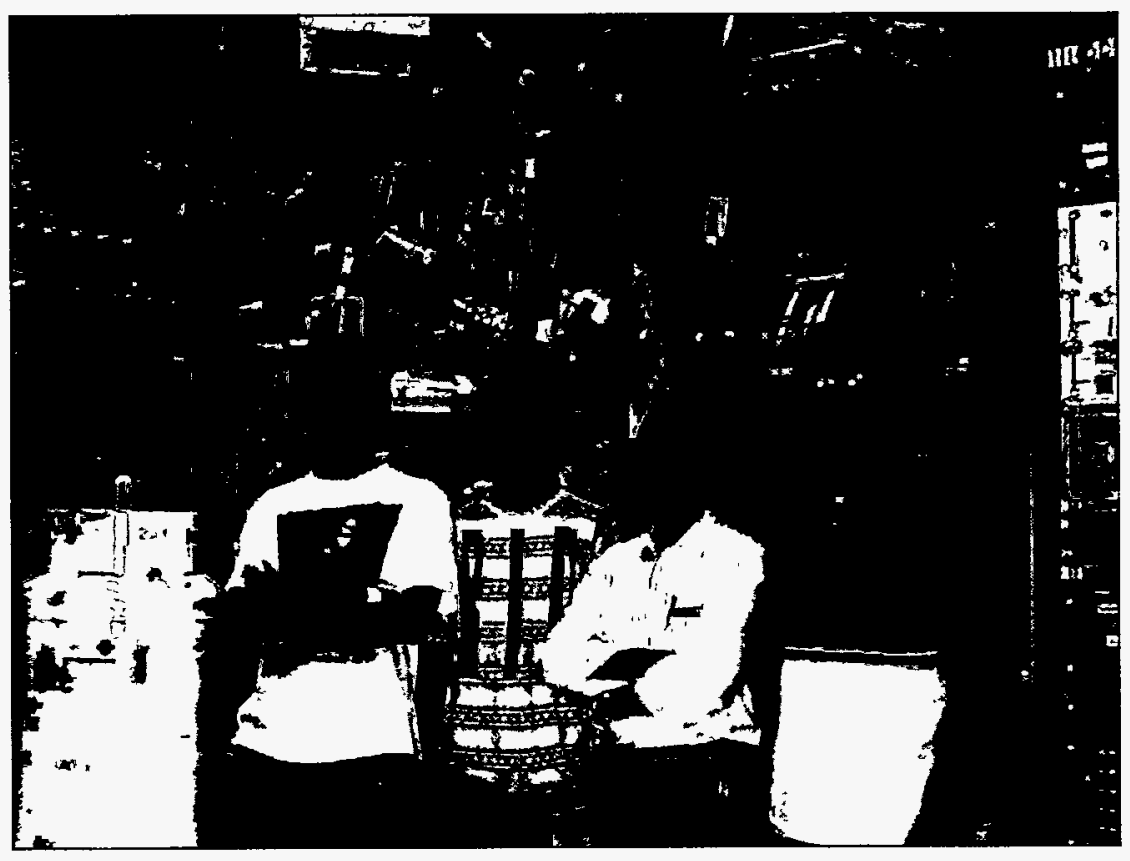

Fig. 3. Pictured are (from left) Stuart Vincet, Chris Pearson, Saeed Mohammadi, Patrick Regan and Bill Gelletly.

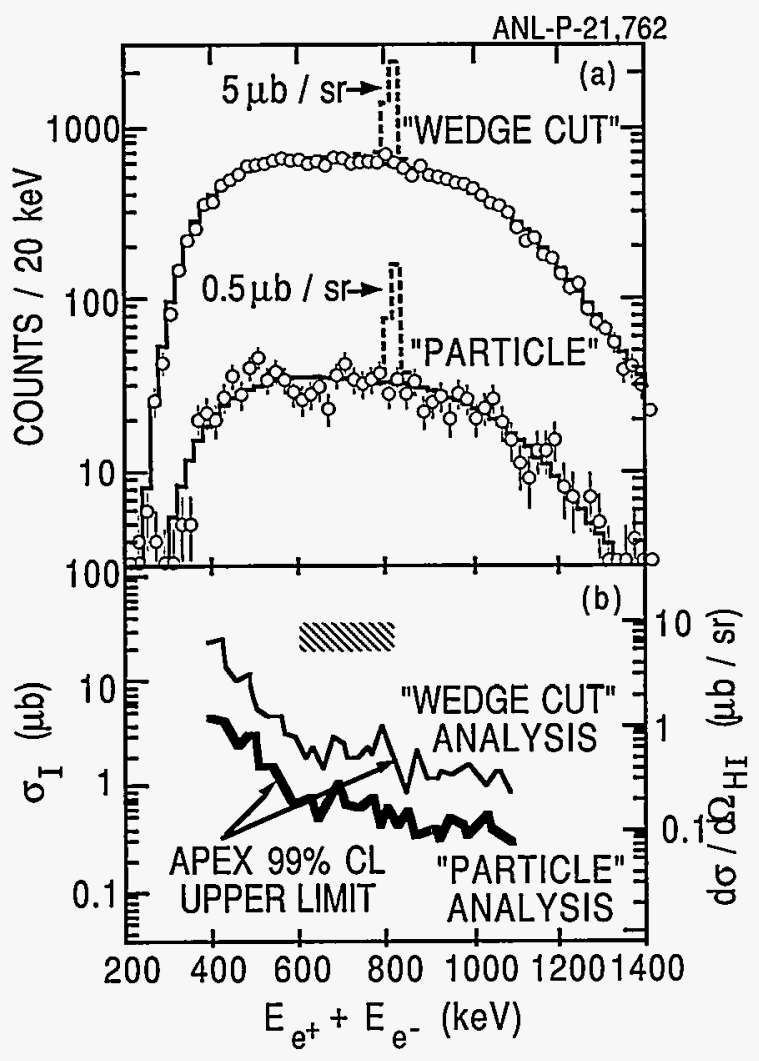

Fig. 4. (a) Positron-electron sum energy spectra analyzed under "wedge cut" and "particle" analyses, from Ref. [1]. The histogram represents the uncorrelated $\mathrm{e}^{+}-\mathrm{e}^{-}$background obtained from event mixing. (b) Production cross section limits derived from the data in (a). The shaded bar shows the range of previously published cross sections. 
uncertainties remain for the M1 case [4]. A recent test of the timing response of the APEX silicon arrays using an avalanche photo-diode (APD) timing source has shown the attainable time resolution to be on the order of 2 to $3 \mathrm{~ns}$. This is good enough to perform detailed $\mathrm{e}^{+}-\mathrm{e}^{-}$angular correlation measurements, and to test the calculations of IPC for magnetic transitions. In-beam measurements to re-examine the question of dynamic positron production in heavy-ion collisions are also planned, where, at beam energies substantially above the Coulomb barrier, strong disagreements are reported between theory and experiment. One possibility is to carry out a systematic measurement of positron yields for several different beam-target combinations at energies up to $9 \mathrm{MeV} / \mathrm{u}$. These, and other experiments are currently under active consideration.

[1] I. Ahmad et al., Phys. Rev. Lett. 75, 2658 (1995).

[2] T. Cowan et al., Phys. Rev. Lett. 56, 444 (1986).

[3] P. Salabura et al., Phys. Lett. 245B, 153 (1990).

[4] P. Schluter et al., Phys. Rep. 75, 327 (1981).

\section{B. TRANSPORT EFFICIENCY OF THE ARGONNE FRAGMENT MASS ANALYZER \\ (B.B. Back, D.J. Blumenthal, C.N. Davids, D.J. Henderson, R.H. Hermann, C.L. Jiang, H.T. Penttila, and A.H. Wuosmaa, ANL)}

The absolute transmission efficiency for the Argonne Fragment Mass Analyzer (FMA) [1] has been measured for ions of $184 \mathrm{~W}, 208 \mathrm{~Pb}$, and ${ }^{232} \mathrm{Th}$ with energies in the range $\mathrm{E}=20-45 \mathrm{MeV}$. These were obtained as $0^{\circ}$ recoils from Rutherford scattering induced by ${ }^{32} \mathrm{~S}$ beams of 50,80 and $100 \mathrm{MeV}$. The measurements were performed for a range of settings for the FMA in order to determine the acceptance of the instrument (defined as the observed fraction of the number of mono-energetic particles entering a geometrical aperture) as a function of energy, angle and mass of the incident ion. The results are compared with predictions of the transport code GIOS [2], which agree well with the data. With these data we conclude that the transport efficiency of the FMA is sufficiently well known to support measurements of absolute cross sections with an accuracy of $10 \%$.

The measurements were carried out using the $45 \mathrm{~cm}$ diam. sliding-seal targetchamber in front of the FMA. The FMA was placed at an angle of $5^{\circ}$ with respect to the beam direction in order to avoid the backgrounds from beam scattered off the first anode. Five square apertures were used, a large $4.5^{\circ} \times 4.5^{\circ}$, and four smaller $1.5^{\circ} \times 1.5^{\circ}$ ones to map out any angle dependence within this area. The results from the large aperture are shown in Fig. 5. The elastic target recoils were registered in a position-sensitive Parallel Grid Avalanche Counter (PGAC) followed after a $40 \mathrm{~cm}$ flight path by a $5-\mathrm{cm} \times 5-\mathrm{cm}$ double-sided Si strip detector (DSSD) with sixteen strips in orthogonal directions on the front and back side of the detector. This detector was used to measure the efficiency of the PGAC detector as given by the probability for obtaining a signal in the PGAC for events detected in the DSSD. A total of 456 measurements were performed over a period of four days. Since the beam energies were substantially lower than the interaction barrier in all cases, we assumed Rutherford cross section for the efficiency calculation. The energy and mass dependence of the transport efficiency was obtained performing measurements for different settings of the FMA after selecting a charge state at or near the maximum of the distribution. 
In panel $b$ of Fig. 5 we show the transport efficiency as a function of a combination of all seven data sets obtained in the present study for the $4.5^{\circ} \times 4.5^{\circ}$ entrance aperture. Here $E$ is the energy of the recoiling ion and $E_{0}$ refers to the ion energy for which the FMA is set. We observed good agreement between the different data sets and were thus encouraged to use the combined data set to derive a transport efficiency (solid curves). It is emphasized, however, that these energy acceptance curves apply only to the specific aperture studied in this work. The use of other apertures with different sizes and/or shapes will result in different energy acceptance curves.

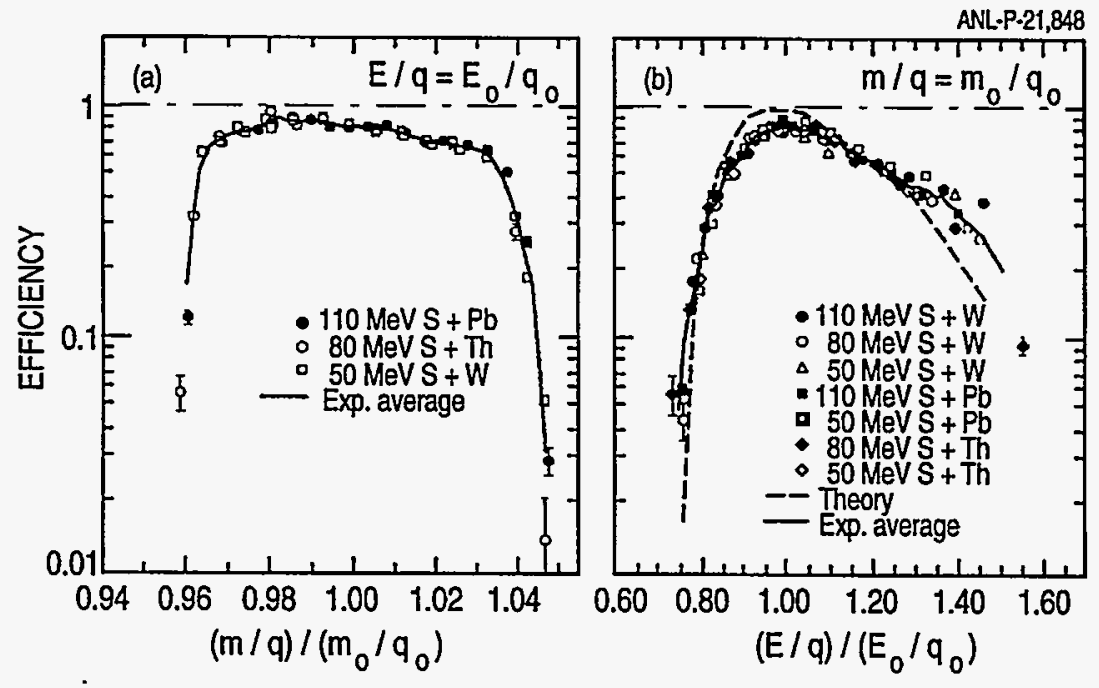

Fig. 5. Measured absolute transport efficiencies for mono-energetic recoil ions (points labeled by the sulfur bombarding energies and targets) as a function of deviation from the central m/q setting, (left) and the E/q setting, $\mathrm{E}_{0} / \mathrm{q}_{0}$ (right) of the FMA with a $4.5^{\circ} \times 4.5^{\circ}$ aperture. A transport efficiency of 1 would mean that all the ions of a given charge state, passing the $4.5^{\circ} \times 4.5^{\circ}$ aperture, were detected. The calculated value of the efficiency as a function of the charge-state setting of the instrument using the code GIOS is shown on the right as a dashed curve.

The transport efficiency of the FMA was also measured as a function of the massto-charge ratio, $\mathrm{m} / \mathrm{q}$, of the incident ion relative to the setting of the FMA i.e.

$(\mathrm{m} / \mathrm{q}) /\left(\mathrm{m}_{\mathrm{o}} / \mathrm{q}_{\mathrm{o}}\right)$. This was done by varying the charge state setting, $\mathrm{q}_{0}$, in steps of $1 / 10$ units for the FMA while the energy setting was kept at the correct energy of the recoils i.e.

$\mathrm{E}_{0}=\mathrm{E}$. The transport efficiency also depends on the ratio $(\mathrm{E} / \mathrm{q}) /\left(\mathrm{E}_{\mathrm{o}} / \mathrm{q}_{\mathrm{o}}\right)$ as shown in Fig. $5 \mathrm{~b}$. The data shown in Fig. 5a are corrected for this dependence such that they represent the dependence on the ratio $(\mathrm{m} / \mathrm{q}) /\left(\mathrm{m}_{\mathrm{o}} / \mathrm{q}_{\mathrm{o}}\right)$ for a fixed ratio $\mathrm{E} / \mathrm{q}=\mathrm{E}_{0} / \mathrm{q}_{0}$. We observed that the FMA acceptance is essentially constant over $\pm 3.5 \%$ from the central $\mathrm{q} / \mathrm{m}$ setting and then falls off rapidly. Thus, we find that the acceptance of the FMA is well understood and recommend that the measured transmission curves be used in a folding procedure with the expected energy (and mass) distributions of evaporation residue in order to obtain reliable absolute cross sections for such processes.

[1] C.N. Davids et al., Nucl. Instr. and Meth. B70, 358 (1992).

[2] H. Wollnik, J. Brezina, and M. Berz, Nucl. Instr. and Meth. A258, 408 (1987). 


\section{COLLECTIVE MOTION IN LIGHT POLONIUM ISOTOPES}

(W. Younes, J.A. Cizewski, H.-Q. Jin, L.A. Bernstein, D.P. McNabb, Rutgers;

C.N. Davids, R.V.F. Janssens, T.L. Khoo, C.J. Lister, D.J. Blumenthal,

M.P. Carpenter, D. Henderson, R.G. Henry, T. Lauritsen, H.T. Penttila, ANL;

D.T. Nisius, Purdue Univ.; and M.W. Drigert, INEL)

The onset of collective motion and the evolution of nuclear shapes near shell closures are still poorly understood. The systematics have recently been studied by Casten, Zamfir, and Brenner [1]. Their work identified a critical phase transition to deformed shapes when $\mathrm{E}\left(2_{1}^{+}\right)<145 \mathrm{keV}$ for nuclei with $38<\mathrm{Z}<82$. The data for $\mathrm{Z}>82$, however, are sparse and do not extend to the neutron midshell $(\mathrm{N}=104)$ where collective effects are expected to be largest.

We have recently measured [2] excited states in ${ }^{194} \mathrm{Po}$, the most neutron-deficient $\mathrm{Z}=84$ isotope studied to date. The ${ }^{194} \mathrm{Po}$ isotope was formed by the $142 \mathrm{MeV}{ }^{28} \mathrm{Si}+170 \mathrm{Yb}$ reaction and the evaporation residues were identified using the ATLAS Fragment Mass Analyzer in coincidence with $\gamma$ rays recorded with 10 Compton-suppressed Ge detectors at the target position. FMA- $\gamma$ and FMA- $\gamma-\gamma$ coincidences were used to identify transitions in 194Po. Figure 6 displays the mass spectrum of the evaporation residues and the deduced level scheme for ${ }^{194} \mathrm{Po}$.
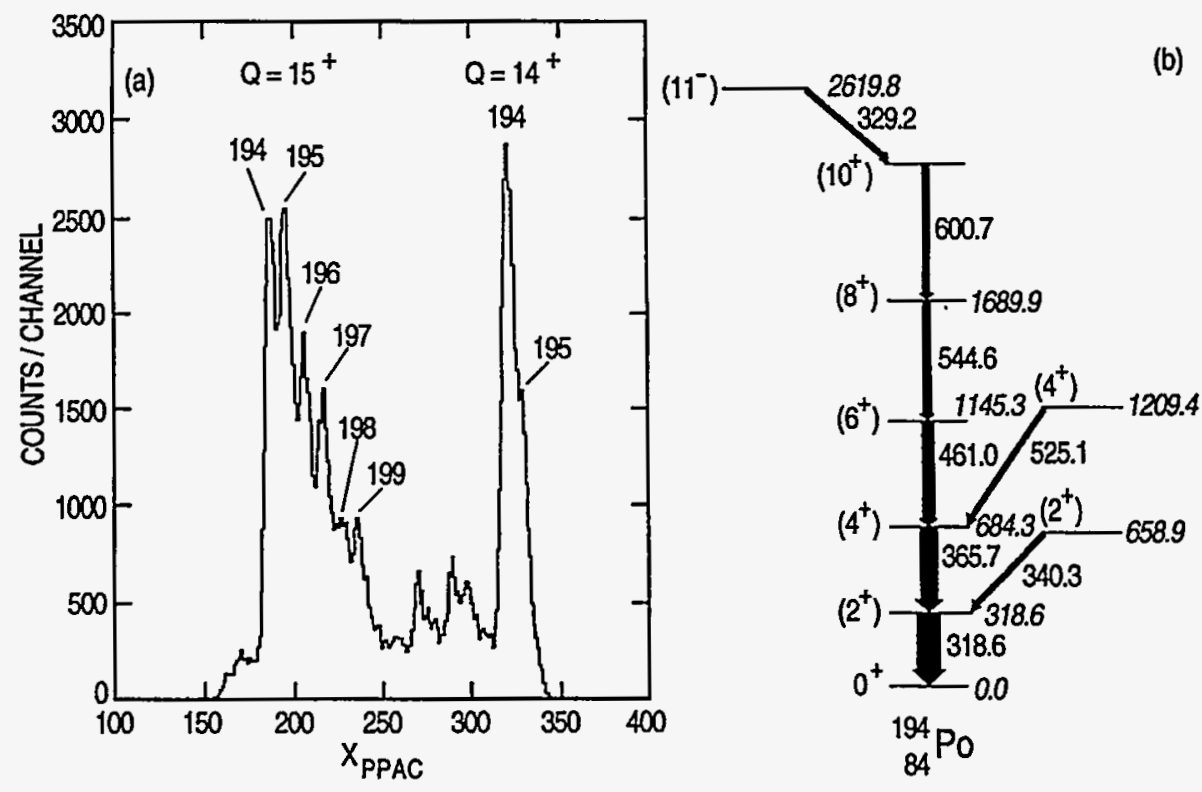

Fig. 6. (a) Projection of the recoil- $\gamma$ matrix onto the position axis, XPPAC. The mass assignments of the peaks are given. (b) Deduced level scheme of ${ }^{194} \mathrm{Po}$.

For the heavier polonium isotopes, the $\mathrm{E}\left(4_{1}^{+}\right) / \mathrm{E}\left(2_{1}^{+}\right)$ratio is always less than 2.00 , the value expected for a harmonic vibrator. ${ }^{196} \mathrm{Po}$ is the first isotope to have clearly collective structure and a shape which is transitional between spherical and deformed. However, the evolution of this shape is very rapid in these lightest polonium isotopes. Extrapolating the change in $2^{+}$energies to $192 \mathrm{Po}$ would predict $E\left(2_{1}^{+}\right) \approx 170 \mathrm{keV}$. Such a low energy is very close to the critical value for the transition to a deformed shape, a 
shape transition which was not previously expected. Experiments are planned for ATLAS to use the FMA in conjunction with the array of Ge detectors to study excitations in ${ }^{192} \mathrm{Po}$.

[1] R.F. Casten, N.V. Zamfir, and D.S. Brenner, Phys. Rev. Lett. 71, 227 (1993).

[2] W. Younes et al., Phys. Rev. C 52, R1723 (1995).

\section{ANGULAR CORRELATION MEASUREMENTS FOR ${ }^{12} \mathrm{C}$ (g.s.)+ ${ }^{12} \mathrm{C}(3-9.64 \mathrm{MeV})$ INELASTIC SCATTERING}

(A.H. Wuosmaa, D.J. Hofman, V. Nanal, B.B. Back, R.R. Betts, D.J. Blumenthal, S. Fischer, M. Freer, B.G. Glagola, D.J. Henderson, R.V.F. Janssens, C.J. Lister, ANL; and D. Nisius, Purdue Univ.)

The study of non-statistical features in the excitation functions of light-heavy ion scattering attracted a great deal of attention some years ago, with the identification of strong, narrow resonances, and their association with quasi-molecular configurations in the composite system. Particular attention has been paid to the ${ }^{12} \mathrm{C}+{ }^{12} \mathrm{C}$ system, where a multitude of resonances have been identified. Many of these states have been linked to extended, deformed cluster configurations in ${ }^{24} \mathrm{Mg}$ at high excitation energy, but reaction models are often able to reproduce at least the qualitative features of the data. Progress in understanding the nature of resonances in this and other systems was slow, however, in part due to the lack of precise experimental information. For instance, many resonances in the ${ }^{12} \mathrm{C}+{ }^{12} \mathrm{C}$ system appear in inelastic reaction channels where the channel spin is nonzero, thus complicating, and, for all intents and purposes, forbidding the extraction of crucial information such as resonance spins. Some progress was made by resorting to particle-gamma-ray angular correlation measurements, however definitive measurements are complicated and without a 4- $\pi$ gamma-detector apparatus, the results are often model dependent and difficult to interpret.

Recent developments in detector technology have renewed the interest in these reactions. Highly segmented silicon strip detectors have been used to study the energy dependence of particle-unbound reaction channels previously experimentally inaccessible, for example mutual inelastic scattering to excited states in ${ }^{12} \mathrm{C}$ which lie above the $\alpha$ particle decay threshold [1,2]. Another opportunity that these detectors provide is to study the angular correlations amongst the alpha decay products from states with non-zero spin to obtain information about the participating angular momenta, and to make spin assignments, for resonances appearing in inelastic channels where one or both of the scattered nuclei is particle unbound.

An excellent example of this situation can be found in the ${ }^{12} \mathrm{C}+{ }^{12} \mathrm{C}$ system, where previous measurements had identified pronounced resonances in the $3-(9.64 \mathrm{MeV})+\mathrm{g}$.s. channel at center-of-mass energies between 28 and $35 \mathrm{MeV}$. This region of excitation energy in ${ }^{24} \mathrm{Mg}$ is particularly interesting, as many other channels display resonant behavior. But without spin information, it is unclear whether the observed features can be related to each other. A resonance-spin measurement, and a study of the alignment of the spin of the excited ${ }^{12} \mathrm{C}$ nucleus, would provide qualitatively new information with which to test both nuclear structure and nuclear reaction models for resonance behavior in this system. Furthermore, since the reaction channel is well matched in angular momentum, the interpretation of the results is somewhat simplified. 
In two measurements, we have used arrays of four, and six, 32-element doublesided silicon strip detectors (DSSDs) to detect the $\alpha$ particles from the decay of the 3-(9.64 $\mathrm{MeV}$ ) level in ${ }^{12} \mathrm{C}$ populated in inelastic ${ }^{12} \mathrm{C}+{ }^{12} \mathrm{C}$ scattering at several energies between 28 and $35 \mathrm{MeV}$ in the center of mass system. The data from the runs with six DSSDs are currently being analyzed, but the earlier data are proving extremely interesting. Shown in Fig. 7a is a reconstructed excitation-energy spectrum for events where three $\alpha$ particles are detected in the strip-detector array, and two of them were observed to emerge from a decaying ${ }^{8} \mathrm{Be}(\mathrm{g} . \mathrm{s}$.$) ; the 3$ - level is clearly evident. Figure $7 \mathrm{~b}$ shows a Q-value spectrum for events where the ${ }^{12} \mathrm{C}$ was detected in its $3-(9.64 \mathrm{MeV})$ level, and in particular the $3-+$ g.s. excitation of interest.

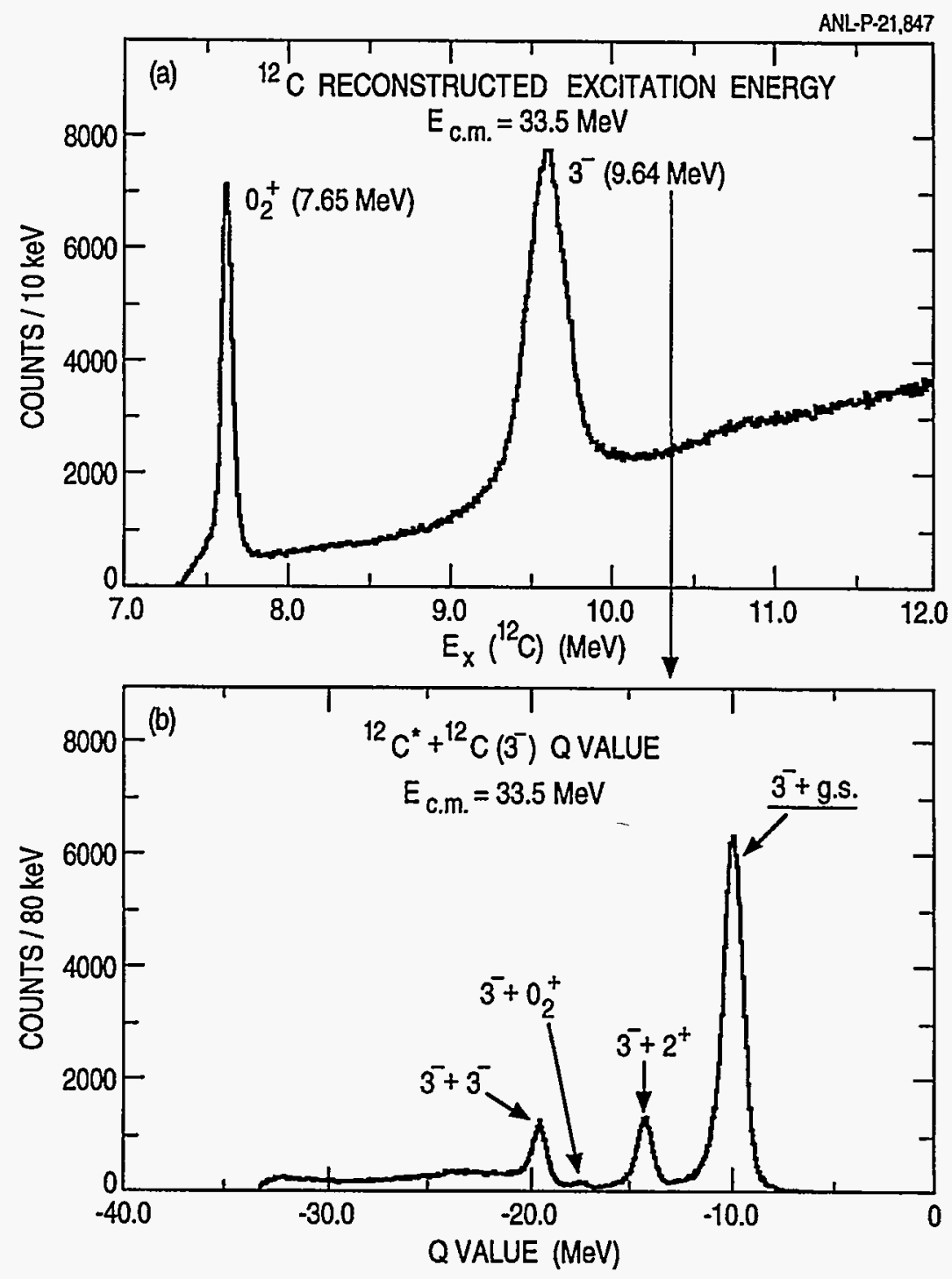

Fig. 7. (a) Reconstructed ${ }^{12} \mathrm{C}$ excitation-energy spectrum from three-alpha particle coincidences, with the requirement that two of the three alpha particles emerge from the decay of a ${ }^{8} \mathrm{Be}$. (b) ${ }^{12} \mathrm{C}+{ }^{12} \mathrm{C} \mathrm{Q}$-value spectrum at $\mathrm{E}$ (c.m.) $=32.5 \mathrm{MeV}$, for events where one ${ }^{12} \mathrm{C}$ is in its $3-(9.64 \mathrm{MeV})$ level. 
Figure 8a shows a matrix of the angular correlation data obtained at an energy of $33.5 \mathrm{MeV}$, the peak of a resonance in the $3-+$ g.s. channel. Here, the abscissa corresponds to the decay angle of the alpha particle in the ${ }^{12} \mathrm{C}$ center of mass frame, measured relative to the beam axis, and the ordinate the center-of-mass scattering angle of the ${ }^{12} \mathrm{C}$. The ridge structure in the correlation matrix contains information about the coupling of the spin of the decaying ${ }^{12} \mathrm{C}$, and the orbital and total angular momentum of the ${ }^{12} \mathrm{C}+{ }^{12} \mathrm{C}$ system. In a simple picture, the slope of the parallel ridges is equal to the ratio of the ${ }^{12} \mathrm{C}$ spin (3) to the orbital angular momentum [3], and a projection of the correlation matrix onto the $\theta$ axis should follow $\left|\mathrm{P}_{\ell}(\cos \theta)\right|^{2}$, where $\ell$ is the orbital angular momentum value. Here, the
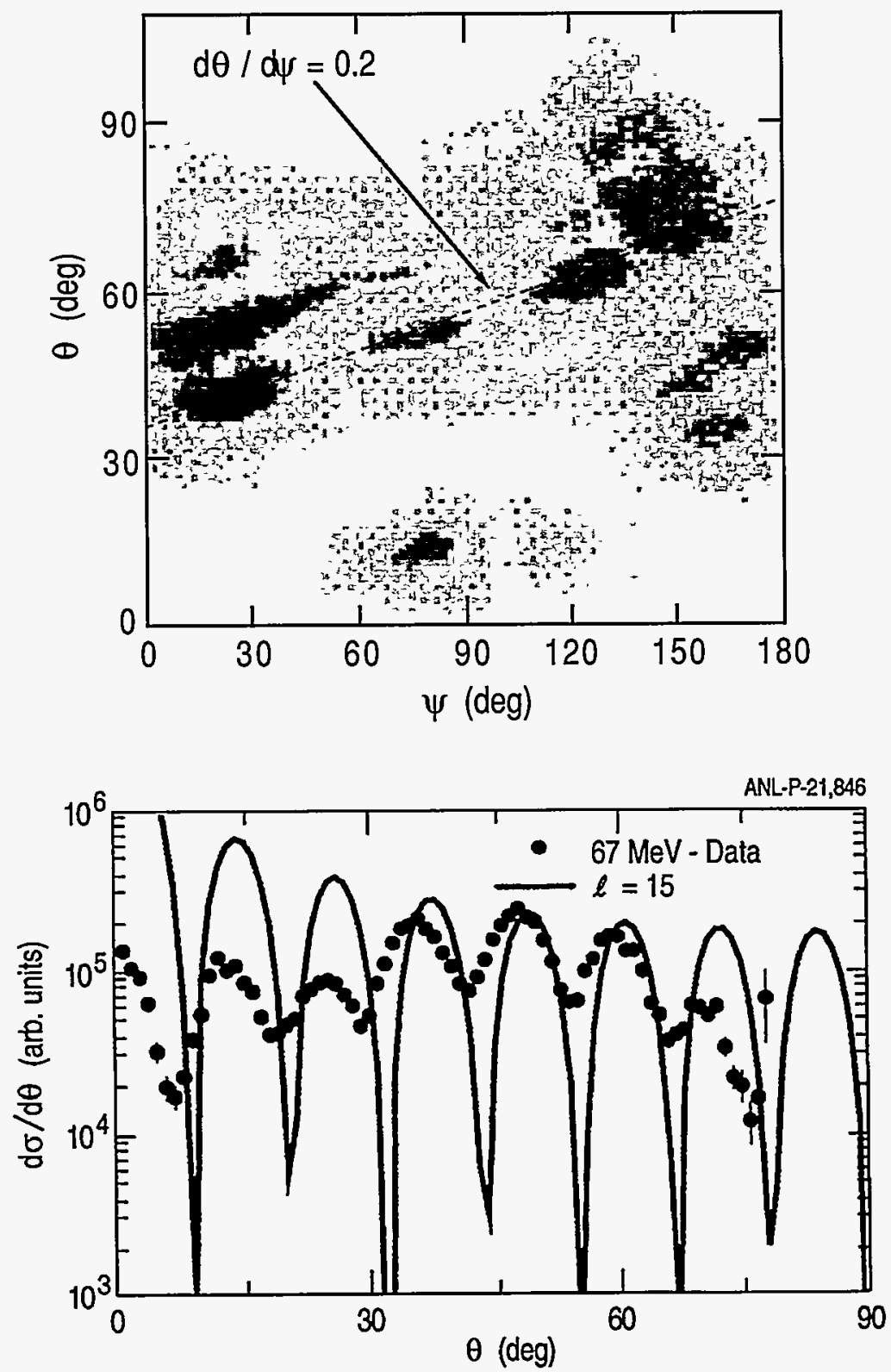

Fig. 8. (a) ${ }^{12} \mathrm{C}+{ }^{12} \mathrm{C}\left(3^{-}\right)$angular correlation matrix at $\mathrm{E}(\mathrm{c} . \mathrm{m})=.33.5 \mathrm{MeV}$. (b) Projection of (a) along the correlation ridges, onto the $\theta$ axis, with $\left|\mathrm{P}_{15}(\cos \theta)\right|^{2}$ (solid curve). 
$\ell$ value is unambiguously identified as 15 (see Fig. 8b). The kinematics of the reaction favor the assumption of an aligned configuration, where the ${ }^{12} \mathrm{C}$ spin and the orbital angular momentum are parallel, a conclusion supported by the alpha-particle angular dependence of the correlation matrix. Under this assumption, we can assign a spin value of 18 to the resonance at $33.5 \mathrm{MeV}$. This value is surprising, as it is in disagreement with various reaction model calculations for resonances in this channel [4,5]. Furthermore, this spin value is inconsistent with the dominant angular momenta deduced for a prominent excitation-function peak at this center-of-mass energy in the angular-momentum mismatched $0_{2}^{+}+0_{2}^{+}$channel $[1,2]$, indicating that while the structures in these two channels appear at the same center-of-mass energy, they most likely arise from very different mechanisms. Analysis of the data is continuing, and a manuscript describing these results is in preparation.

[1] A.H. Wuosmaa et al., Phys. Rev. Lett. 68, 1295 (1992).

[2] A.H. Wuosmaa et al., Phys. Rev. C50, 2909 (1994).

[3] W.D.M. Rae et al., Phys. Lett. 156B, 167 (1985).

[4] Y. Abe et al., Phys. Rev. C19, 1365 (1979).

[5] Y. Hirabayashi et al., Phys. Rev. Lett. 74, 4141 (1995).

\section{E. THE AYE-BALL: THE ARGONNE-YALE-EUROPEAN GAMMA RAY SPECTROMETER}

From August to December 1995 the AYE-ball gamma-ray spectrometer was assembled at the Fragment Mass Analyzer (FMA) to exploit the unique capabilities of the ATLAS and FMA for studying the structure of nuclei far from stability. Rare, but important, nuclides formed at the level of 1 in 100,000 fusion reactions can be isolated and studied. This corresponds to production cross sections of a few microbarns. The device consists of gamma-ray detectors from Strasbourg (France), Daresbury, Liverpool and Manchester (U.K.), from Yale University in the U.S.A., and from ANL, all operated in coincidence with the FMA and suitable focal plane trigger detectors.

The plans for this detector system were developed by W. Gelletly from the University of Surrey, U.K. and C.J. Lister from Argonne National Laboratory. The detailed design work was done at Daresbury Laboratory (U.K.) by J. Simpson and D.D. Warner and by D.J. Blumenthal, C.N. Davids and J. Falout at Argonne. The project has been an international effort from the outset and fifteen separate experiments have been performed, with participation of more than forty scientists from thirteen countries.

The gamma ray spectrometer (Fig. 9a) consists of nine $>70 \%$ efficient $\mathrm{HpGe}$ detectors and ten $>25 \%$ detectors, all Compton suppressed, which can be augmented by 5 planar LEPS x-ray detectors when needed. It forms a detector system which is $1.2 \%$ efficient for detecting $1.33 \mathrm{MeV}$ gamma ray photopeaks, with a peak-to-total ratio of 53\%. It is intermediate in efficiency between most "first" and "second" generation spectrometers. Consequently, as a standalone device it is not particularly unique. However, it is its operation with a very sensitive triggering system which makes it unique and allows studies which could not be done at the world's largest gamma-ray facilities; Gammasphere, GASP, or Eurogam. 

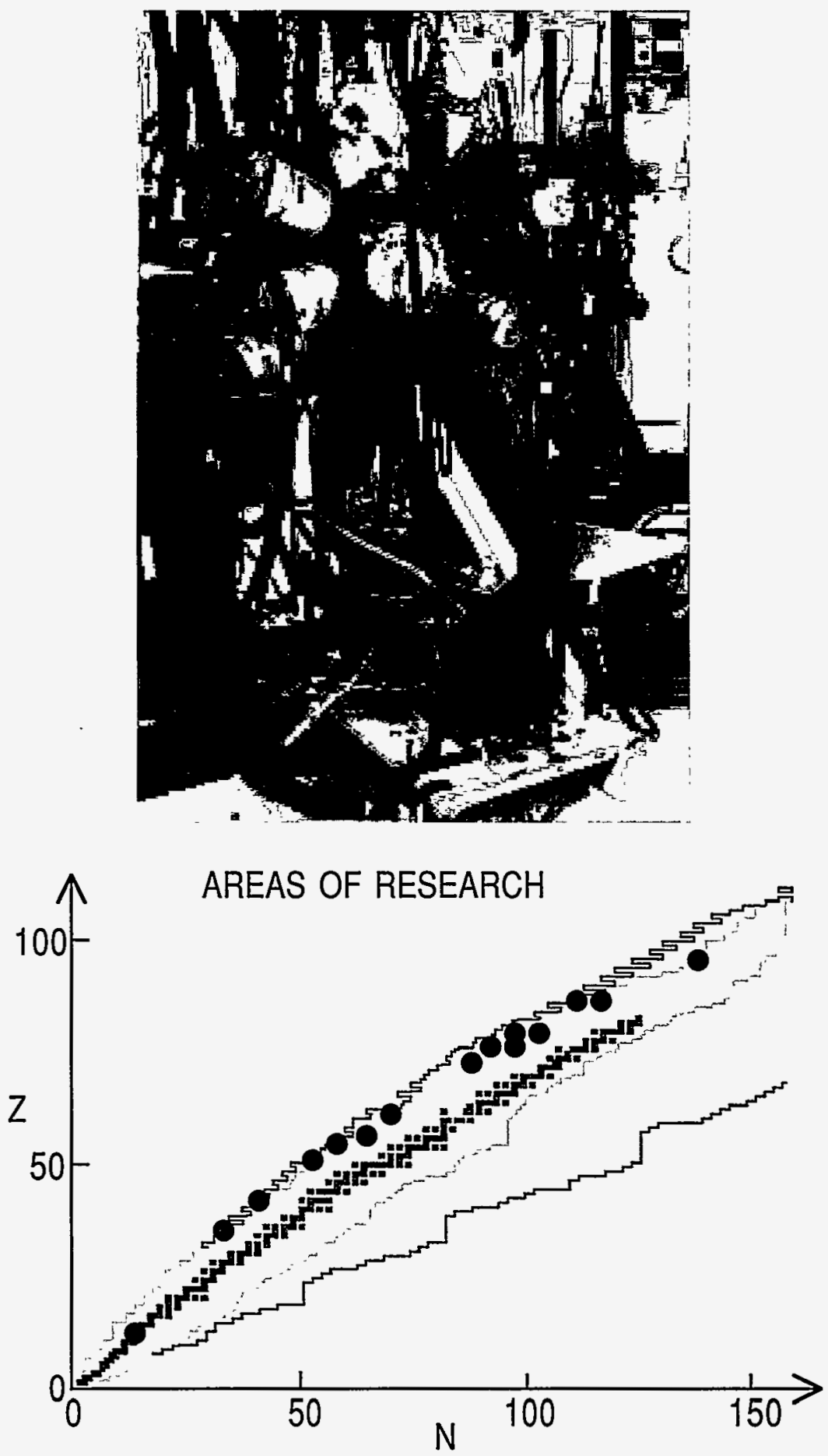

Fig. 9. The top portion shows the AYE-ball used at the target position of FMA for $\gamma$-ray experiments. It consists of nine $70 \% \mathrm{Ge}$ and ten $25 \%$ Ge detectors. The bottom figure shows the nuclides studied (solid circle) by this device in conjunction with the FMA during July-December 1995 ATLAS schedule. The studies spanned the mass range $A=24$ to 226 . 
Triggering comes from four independent sources: neutron detection, mass measurement of the residues, the characterization of the stopping, or the radioactive decay of the ions. It is the simultaneous measurement of these quantities which allows excellent channel selection and thus permits individual gamma-ray transitions to be associated with fully identified nuclei.

Experiments have been done on the spectroscopy of light, intermediate and heavy nuclei from mass $A=24$ to 226 , focusing on shell stabilization of spherical, prolate, oblate and octupole deformed shapes and on the properties of quantum systems which are particle-unbound (Fig. 9b). A series of experiments involving "double" nuclear reactions has been investigated, in which a beam of particles strikes a target producing a secondary beam of radioactive nuclei which are then used to induce further nuclear reactions on another target.

\section{RESEARCH RELATED TO THE CONCEPT FOR AN ADVANCED EXOTIC BEAM FACILITY BASED ON ATLAS}

A working paper entitled "Concept for an Advanced Exotic Beam Facility Based on ATLAS" was prepared in early 1995 and used in presentations at the town meetings associated with the Long Range Plan for Nuclear Physics in the United States. The Long Range Plan, which is currently being finalized by NSAC, gives high priority to the construction of a cost-effective, ISOL-based, advanced facility for doing nuclear physics with accelerated radioactive beams. A progress report on the concept for an ATLAS-based facility was given in the May, 1995 Newsletter. To further develop the case for such a facility and to be ready for a possible call for proposals by the DOE in the not-too-distant future additional research is in progress. The R\&D described below is all related to the accelerators or facilities; there are also nuclear physics experiments with radioactive beams in progress at ATLAS, such as the ${ }^{18} \mathrm{~F}(\mathrm{p}, \alpha)$ measurements reported on in the last newsletter.

A half-scale, cold model of a Radio-Frequency Quadrupole accelerator being designed for radioactive beams with very low charge states has been constructed and is currently undergoing tests (see Fig. 10). Measurements of its resonant frequency, Q, and shunt impedance yielded values very near expectations. The next step is to measure the rf voltage limits of the model by driving it with if power at a low duty factor. This test will be carried out at NSCL using the rf transmitter of the K500 cyclotron since adequate power at $20 \mathrm{MHz}$ is not currently available at ATLAS. Once the voltage limit of the model is determined the design of the full sized RFQ will be completed and the first module constructed. This work will continue through FY1996.

Another component required for the radioactive beam accelerator is a high-gradient superconducting quadrupole. This focusing element would be used in the low-charge-state superconducting injector linac which would accelerate ions with $\mathrm{m} / \mathrm{q}$ up to 66 from initial velocities of less than $0.01 \mathrm{c}$. Superconducting resonators for this velocity range are the type currently in use in the ATLAS positive-ion injector (PII) and require no further development. The lower charge states in the radioactive beam injector compared to those currently in use in PII require much stronger transverse focusing elements, however. Hence, we are in the process of developing a prototype quadrupole with a gradient in the 


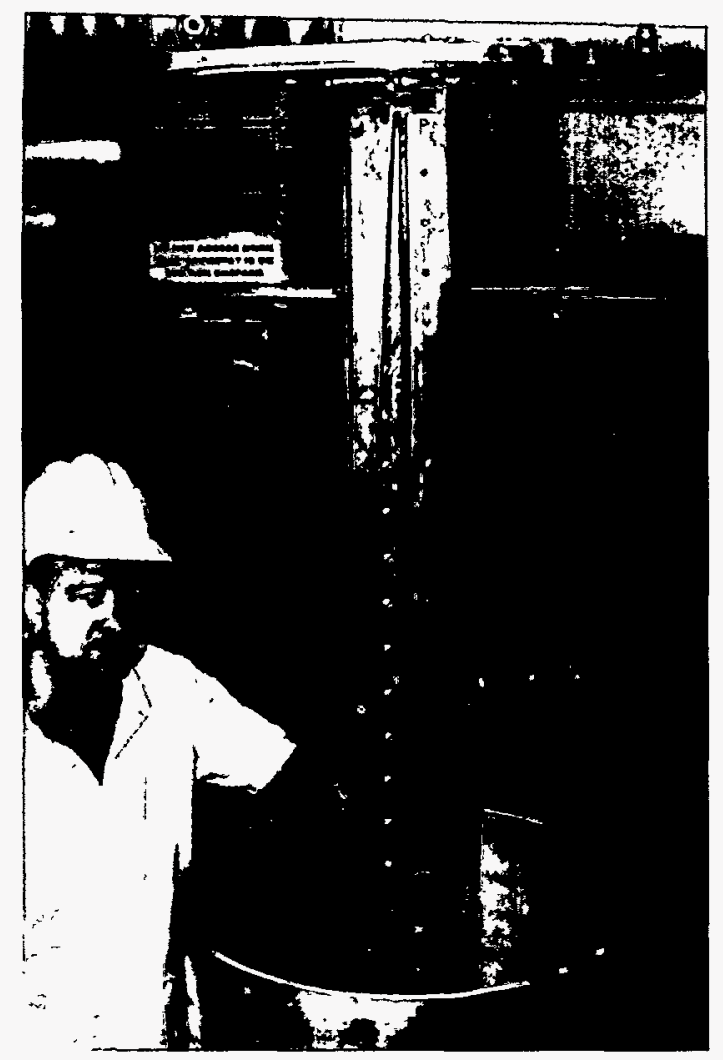

Fig. 10. Photograph of the half-scale RFQ cold model. The structure is a "split-coax" type of resonator; two of the RFQ vanes are supported from one end of the structure and the other two from the other end. In this photograph the structure is opened to illustrate two vanes hanging from an end flange at the top of the figure and two just visible inside the tank at the bottom.

range of 300-350 T/m. A preliminary design with a bore diameter of $3 \mathrm{~cm}$ has been worked out and two prototype coils have been wound and tested commercially up to currents which correspond to gradients of $280 \mathrm{~T} / \mathrm{m}$. This performance level could be used in the low-charge-state injector, but somewhat higher gradients would be optimal. Further work on the prototype design will be carried out during 1996.

During the summer of 1995 Jeff Wendlandt, a graduate student in the Electrical Engineering and Computer Science Department at U. C. Berkeley, worked at ANL and prepared a resource paper entitled "Robotics and Remote Systems Report for the Exotic Beam Facility." This paper summarizes the capabilities of many commercially available remote handling systems as well as conversations which he had with several experts at national laboratories and universities. Systems for remote handling via telerobotics methods, such as the Monitor system in use at LAMPF, will be necessary in the target/ion source modules of a high power radioactive beam facility. It will be very important for our designs to build upon the experiences accumulated at meson factories such as LAMPF, TRIUMF, and PSI. Containment and handling methods used in Hot Cells such as the Alpha/Gamma facility at Argonne will also be important in radioactive beam facilities. 
The design parameters and the overall facility performance depend critically on the performance of the ion sources for the radioactive ions. Critical parameters are their ionization efficiencies, delay times (for short-lived radionuclides), emittances, energy spreads, and chemical selectivity. Much work with ISOL-type ion sources has been done over the past 20 years or so, but R\&D in this field is still active around the world. There is still the need for further detailed and quantitative measurements of the parameters of the various types of ion sources which are candidates for use in an advanced ISOL-type radioactive beam facility. We have recently begun an R\&D program at ATLAS to measure some of the parameters which are important to our radioactive beam initiative. This program has two aspects; first, the conversion of the tandem negative-ion source test stand for studies of ISOL-type sources, and, second, studies of efficiencies and release times of neutron-rich fission products. A high temperature plasma ion source has been obtained from Ron Gill at BNL and is now operational at the ATLAS ion source test stand. The old emittance measuring apparatus at this test stand is currently being refurbished (modernized) to study and optimize the optics of this type of ion source.

The second type of ion source R\&D mentioned above will be carried out at the existing Physics Division Dynamitron Facility. The plan is to use $3.5 \mathrm{MeV}$ deuterons from the Dynamitron to produce secondary neutrons via the $\mathrm{d}+\mathrm{Be}$ reaction. The beryllium target will be within $5 \mathrm{~cm}$ of a uranium sample in the hot plasma ISOL-type ion source. Short-lived fission fragments, such as $1.2 \mathrm{sec}{ }^{142} \mathrm{Xe}$, will be ionized and extracted from the source. A shielded cave to be constructed in the Dynamitron experimental area has been designed and will be assembled this winter. In the meantime safety documentation related to operating the Dynamitron in this mode is being prepared and reviewed.

The Dynamitron will also be used in the near future for another measurement related to our exotic beam proposal. Our proposal involves initial acceleration of $1^{+}$ radioactive ions in the RFQ discussed above, followed by stripping to 2 or $3+$ at about 8 $\mathrm{keV}$ per nucleon. There are limited data in the literature on stripping such low velocity ions in helium gas. These data show anomalously high efficiencies, over $45 \%$ into the $2^{+}$ charge state in some cases. We plan to study in more detail the stripping of $\mathrm{Xe}^{1+}$ ions in helium gas as a function of gas pressure and beam energy for 1 to $2 \mathrm{MeV}$ beams. The Dynamitron can provide Xe beams in this energy range and existing apparatus, previously used for Coulomb explosion studies of molecular ions by the atomic physics group, is available for these measurements.

\section{PROGRAM ADVISORY COMMITTEE}

The next ATLAS PAC meeting will be held on Thursday, January 18 and Friday, January 19, 1996 with proposal presentations. The PAC expressed the wish to have written proposals supplemented with oral presentations in order to facilitate information transmittal and to help clear up questions and concerns. Spokespersons or their designates will present their proposals to the PAC on Thursday, January 18. The presentations should briefly summarize the written proposal in 10 minutes, with 5 minutes set aside for questions and discussion with the Committee.

The presentations are requested for the benefit of the PAC - if for some reason a presentation is not feasible, the Committee will proceed, as in the past, on the basis of the written material. The presentations will be open to all those interested. 
Thursday evening we plan a dinner reception for all participants - this may provide a forum for discussion to continue on a more informal basis.

The last ATLAS Program Advisory Committee meeting was held on May 26 and 27,1995 . The PAC reviewed 28 proposals requesting 133 days running time and recommended 23 proposals (11 from outside user spokespersons) for 101 days.

The present Program Advisory Committee members are:
Russell Betts
David Church
David Fossan
Paul Kienle
Mark Riley
Michael Wiescher
Frank Wolfs
ANL
Texas A\&M University
SUNY @ Stony Brook
T. U. Muenchen
Florida State University
University of Notre Dame
University of Rochester

The Committee is chaired by John Schiffer.

\section{Summary of the May 1995 ATLAS PAC Meeting}

- Number of new proposals submitted

- Total number of days requested in the proposals for this period

- Total number of proposals approved

- Total number of days recommended

- Active backlog of approved beam time (in days) prior to the PAC meeting

- Number of scientists involved in proposals submitted to the May 1995 PAC 131

- Number of institutions represented

- Number of countries represented

\section{LIST OF PROPOSALS APPROVED AFTER THE MAY 1995 PAC MEETING}

Exp. Spokes-

No. person

433-3 Fendrich

503-4 Rehm
Title

Days Approved

Heavy Ion Irradiation of High Temperature

Superconductors

Study of $18 \mathrm{~F}(\mathrm{p}, \alpha)$ and ${ }^{18} \mathrm{~F}(\mathrm{p}, \gamma)$ Reactions using a Radioactive ${ }^{18} \mathrm{~F}$ Beam 
521-2 Conticchio

Single-Particle Energies and Proton-Neutron Interactions near Sn-100: Study of the Decay of $105,106,107 \mathrm{Sb}$

524-2 Cizewski

g-Factor Measurement of a High Spin M1 Band in ${ }^{197} \mathrm{~Pb}$

527-2 Fischer

Coulomb Excitation of Radioactive ${ }^{79} \mathrm{Rb}$ : Phase 2

528-3 Dunford

Polarimeter for Metastable $\mathrm{Ar}^{17+}$ Ions

530-2 Livingston

Atomic Spectra and Lifetimes in Highly Charged Krypton Ions

535-3 Ali

Spectral Distributions of the Two-Photon Decay of $\mathrm{Kr}^{34+}$

553-2 Hofman

Survival Probability of Th-Like Fragments - Energy

Dissipation in Hot Nuclei

558-2 Khoo

Coulomb Excitation of 232Th - Exploring an Unconventional Approach of Projectile Excitation with a Thick Target

$561 \quad$ Paul

Identification of $\gamma$-Ray Transitions in the Neutron-Deficient ${ }_{51}^{110} \mathrm{Sb},{ }_{53}^{110} \mathrm{I}$, and ${ }_{54}^{110} \mathrm{Xe}$ Isobars

$562 \quad$ Davids

A Search for Spontaneous Proton Emission from ${ }^{189}$ At and ${ }^{196} \mathrm{Fr}$

563 Wiescher

Lifetime Measurements of the $\mathrm{N}=\mathrm{Z}$ Nuclei ${ }^{80} \mathrm{Zr}$ and ${ }^{84} \mathrm{Mo}$ for the Study of rp-Process Nucleosynthesis at X-Ray Burst Conditions

564 Batchelder

The Behavior of Intruder Based States in Light Bi and Tl Isotopes: Study of the $\alpha$ Decay of ${ }^{187} \mathrm{Bi}$

566 Zhang

Investigating Higher-Seniority $\left(v h_{11 / 2}\right)^{n}$ Excitations in $119-125 \mathrm{Sn}$

567 Wuosmaa

Angular Correlation Measurements for ${ }^{12} \mathrm{C}\left({ }^{12} \mathrm{C},{ }^{12} \mathrm{C}\right){ }^{12} \mathrm{C}\left(3^{-}\right)$ Inelastic Scattering

$568 \quad$ Reviol

Target Tests for Transient Field g-Factor Measurements in ${ }^{184} \mathrm{Pt}$ and ${ }^{180} \mathrm{Pt}$

$570 \quad$ Sanders

Experimentally Deduced Fission Barrier Energies for ${ }^{48} \mathrm{Cr}$

4

$571 \quad$ Ahmad

In-Beam Spectroscopy of ${ }^{224} \mathrm{U}$

572 Henning

Search for the New Element $Z=112$ via the Reaction ${ }_{30}^{70} \mathrm{Zn}+{ }_{82}^{208} \mathrm{~Pb}$ 
574 Rehm Study of the Single-Particle States Around N,Z=28

with a Radioactive $56 \mathrm{Ni}$ Beam $\quad 9$

577 Seweryniak In-Beam Spectroscopy of ${ }^{103} \mathrm{Sn}$

\section{ATLAS USER GROUP EXECUTIVE COMMITTEE}

The current members of the ATLAS User Group Executive Committee are:

$\begin{array}{ll}\text { Partha Chowdhury } & \text { University of Massachusetts, Lowell } \\ \text { John Fox } & \text { Florida State University } \\ \text { Lee Riedinger } & \text { University of Tennessee } \\ \text { Frank Wolfs (Chair) } & \text { University of Rochester }\end{array}$

Frank Wolfs can be reached on INTERNET at wolfs@nsrl31.nsrl.rochester.edu. Users are encouraged to communicate with the Executive Committee about ATLAS issues. The location of the ATLAS World Wide Web pages (through the ANL Physics Division pages) is http://www.phy.anl.gov/.

The Executive Committee held an ATLAS User Group Meeting at the fall DNP meeting in Bloomington, Indiana, this past October that was attended by about 42 scientists. John Fox chaired the meeting and presented the opening and User's Perspective of the ATLAS Program. Walter Henning then discussed several institutional issues concerning an exotic beam facility and the cycle of Gammasphere to ATLAS. Jerry Nolen summarized recent performance and the status of ATLAS and also described current accelerator R\&D related to the exotic beam proposal. Cary Davids presented a review and preview of the intensive schedule of the FMA covering the AYE-ball experiments that dominated the schedule from August -December. John Schiffer concluded the meeting with a discussion of PAC matters detailing the proposal deadline, meeting dates and structure of the next PAC meeting.

The Executive Committee reports that so far this year it has received only one complaint from an ATLAS User. Please do not hesitate to contact the User Executive Committee if you have comments, questions, and/or suggestions concerning the operation of ATLAS.

The ATLAS User Group Executive Committee is currently working on a revised version of the ATLAS Users Handbook. The last edition of the handbook is now five years old. We are also considering better ways of distributing information about the actual operation of ATLAS to the Users. One possibility is to request at the end of each experiment from the spokesperson a one paragraph summary of the run which includes reaction information, beam currents delivered, beam timing, and any other information that might be useful to other users. This information can then be made available to the ATLAS Users on a monthly basis via email or via the ATLAS World Wide Web pages. 